\title{
Más allá de la teoría económica dominante: diálogos entre la economía feminista y la economía ecológica
}

\author{
Marina CHECA \\ Universidad de Granada \\ marinacheca@correo.ugr.es \\ Consuelo DíAZ \\ Universidad de Granada \\ $\underline{\text { cdiaz81@correo.ugr.es }}$
}

Received: $14 / 11 / 2015$

Accepted: $30 / 12 / 2015$

\begin{abstract}
Resumen
Resulta evidente la incapacidad de la teoría económica dominante para dar respuesta a las necesidades humanas y para sostener la vida en su conjunto dentro de los límites biofísicos del planeta. La economía convencional, lejos de reflexionar sobre las diferentes dimensiones que conforman la sostenibilidad global, se basa en la obtención de beneficio y en el crecimiento económico centrando su análisis en la esfera monetizada. Por ello, es necesario ampliar nuestra mirada más allá de los mercados, abriendo nuevos caminos de reflexión y debate en torno a otras corrientes críticas económicas. En este artículo se propone entablar un diálogo entre la economía feminista y la economía ecológica, repensando los puntos de encuentro entre ambas perspectivas, para reflexionar entorno a una economía que tenga en cuenta el trabajo de las mujeres y la naturaleza.
\end{abstract}

Palabras clave: teoría económica dominante, economía feminista, economía ecológica, sostenibilidad, justicia social, medio ambiente, trabajo de cuidados

\begin{abstract}
It turns out to be clear the incapability of the domineering economic theory to give answer to the human needs and to support the life in its set inside the biophysical limits of the planet. The conventional economy, far from reflecting about the different dimensions that shape the global sustainability, it is based on profits obtaining and on the economic growth centering its analysis on the monetized sphere. Therefore, extending our look beyond the markets is required, opening new ways of reflection and debate concerning other economic critical currents. In this article it is proposed to initiate a dialogue between feminist economy and ecological economy, reconsidering the meeting points between both perspectives, to reflect about an economy that takes into consideration the women work and the nature.
\end{abstract}

Key-words: Domineering economic theory, feminist economy, ecological economics, sustainability, social justice, environment, care work

JEL Classification:B00, B54, Q57

\section{Introducción}

El discurso económico dominante acerca de la riqueza y su medición, del proceso económico y de la organización y progreso de la sociedad basada en las relaciones de producción capitalistas, ignora todas las actividades que se encuentran al margen de la esfera mercantil. Así, el patrimonio natural y los trabajos relacionados con los cuidados de las personas, imprescindibles para el mantenimiento de la vida, son soslayados, de manera sistemática. La vida, y el subsistema económico, que forma parte de ella, no son posibles sin los bienes y servicios proporcionados por el planeta (limitados y en proceso de deterioro) y sin los trabajos de cuidados. La supervivencia de las sociedades humanas depende de estas tareas esenciales para la sostenibilidad de la vida, pero invisibles para el discurso económico dominante (Amoroso, Bosch y Fernández 2003).

En este trabajo se propone profundizar en estas dos dimensiones a partir de los aportes teóricos planteados desde el enfoque de la economía ecológica y el enfoque de la economía feminista. Se trata de entablar un diálogo entre ambas perspectivas teóricas que refleje la importancia de ampliar sus miras y plantear relaciones entre ambas disciplinas, que permitan indagar otras 
formas de analizar los sistemas económicos y construir otras formas de entender el mundo desde la equidad, la justicia social y ecológica.

Para llevarlo a cabo, el estudio se divide en cuatro partes. En la primera, se trata de poner de manifiesto que el paradigma económico dominante ha dejado al margen las dimensiones ecológicas y de género, consolidándose como ciencia económica en el universo aislado de los valores monetarios. En la segunda y tercera partes, se presentan las aportaciones de los enfoques feminista y ecologista a la teoría económica. En la cuarta parte se buscan los puntos de encuentro entre ambos enfoques para proponer posibles líneas de investigación futuras. Finalmente, se plantea el paradigma de la sostenibilidad de la vida para recorrer nuevos caminos y construir otras formas de organización económica y social de las poblaciones humanas superando el sesgo antropocéntrico y androcéntrico del paradigma económico dominante.

\section{La ausencia de las dimensiones ecológica y de género en el paradigma económico domi- nante}

Desde el origen, la ciencia económica se ha construido sobre una invisibilización de todas las tareas realizadas para la reproducción de la vida y sobre una conciencia de dominio y explotación de la naturaleza. Con la transición del feudalismo al capitalismo, la economía inicia su andadura como ciencia y se inserta el método científico al estudio de la actividad. La obra por excelencia de este período es La riqueza de las naciones cuyo autor, Adam Smith, defensor del liberalismo económico, analiza de manera rigurosa el funcionamiento de los mercados.

El pensamiento económico clásico surge en el contexto del proceso de industrialización, momento en el que comienza a difuminarse la frontera entre el subsistema de producción de bienes y servicios y el subsistema de reproducción de las personas (Cabrera y Escobar 2014, p. 224). En efecto, como establece Mayordomo (2000) Smith y demás pensadores clásicos ejercieron una notable influencia sobre la consolidación de una visión (parcial) de "lo económico" que margina a las mujeres y a las actividades que éstas realizan en el campo de estudio de la economía. Con todo, reconocían la importancia del trabajo doméstico y de cuidados realizado en los hogares como garantes de la reproducción de la mano de obra (Carrasco 2006, p. 34). Al definir el salario en términos de subsistencia, admitían el vínculo existente entre la producción de bienes en el mercado y la reproducción de la fuerza de trabajo (Pérez 2005, p. 38).

También se reconoce la importancia de las aportaciones de las mujeres a la historia de pensamiento político y económico a lo largo de los siglos XIX y XX. ${ }^{1}$ Autoras como Jane Marcet, Harriet Martineau, Harriet Taylor Mill, Rosa Luxemburgo o Joan Robinson, entre otras, han contribuido a las discusiones y los planteamientos sostenidos en torno a las ideas de los economistas clásicos en lo que se refiere al trabajo y la situación de las mujeres ${ }^{2}$ Pero, al cabo, el pensamiento clásico no llega a situar el trabajo realizado por las mujeres en el marco analítico adecuado. Con la economía neoclásica desaparecerá esta preocupación por incorporar al análisis económico el trabajo realizado por las mujeres en el ámbito familiar (Carrasco 2006, p. 37).

En la década de 1870, la escasa sensibilidad de los economistas clásicos para observar los procesos sociales más allá de la producción industrial (Carrasco 2012, p. 31) se vuelve aún más profunda con el surgimiento del marginalismo, punto de partida del pensamiento neoclásico. El objeto de estudio se desplaza de la producción de riqueza al mercado, con lo que la producción realizada en los hogares queda relegada a la absoluta invisibilidad. Se parte del equilibrio automático del mercado y del análisis marginal en las decisiones individuales, donde los consumidores actúan de forma racional para maximizar su utilidad personal. Se asume la concepción del individuo como homo xeconomicus ${ }^{3}$ cuyo comportamiento está basado en el interés propio bajo

\footnotetext{
1 El exhaustivo estudio realizado por Perdices y Gallego (2007) en torno a las aportaciones de las mujeres a la ciencia económico nos permite no entrar en más detalle en esta línea de investigación.

2 Veánse también los trabajos de Gallego (2000, 2010, 2011).

3 Sujeto que propugna la teoría neoclásica que actúa siempre racionalmente. Se ignora cómo se reproduce, porque siempre llega crecidito, aseado, bien vestido y a su hora a las puertas de una empresa.
} 
un deseo insaciable de adquirir y acumular, sin manifestar relaciones emocionales a través de una conducta autónoma. Se ignora que tras cada homo æeconomicus hay una mujer, pero ésta es desplazada al ámbito de la subordinación y sumisión (Tello, 2005, p. 61). Esta división sexual del trabajo ${ }^{4}$ que relaciona al hombre con la esfera económica genera una desigual valoración social de la mujer al reducir sus tareas al ámbito de la reproducción, dependiente de los recursos masculinos provenientes del mundo monetizado (Cabrera y Escobar 2014, p. 224).

En cuanto a la dimensión ecológica, pese a que algunos economistas clásicos pusieron de manifiesto la existencia de límites al crecimiento, estas preocupaciones fueron abandonadas paulatinamente. ${ }^{5}$ Siguiendo a Naredo (1996), el razonamiento económico se separa completamente del mundo físico, lo que se traduce en un divorcio definitivo entre la economía y el medio ambiente. El mercado se eleva a factor principal para el crecimiento económico y la naturaleza se concibe como fuente inagotable de recursos. En definitiva, la invisibilidad del trabajo realizado por las mujeres descrita con anterioridad se acompaña de esta subvaloración de los recursos naturales, en aras de favorecer la productividad en la creación de riqueza en las naciones.

El sistema socioeconómico que impera en la actualidad está sustentado sobre el paradigma neoclásico, que coloca a los mercados en el centro de la organización social y prioriza la lógica de acumulación de capital sobre la lógica de la vida humana y no humana. Se considera a la naturaleza como un input más para la creación de riqueza (Pérez 2014, p. 37), como una fuente de ingresos, separada de la humanidad absolutamente mercantilizada, siguiendo un esquema extractivista. ${ }^{6}$

En definitiva, la teoría neoclásica, que hoy sustenta el discurso político neoliberal, adolece de fuertes sesgos androcéntricos y antropocéntricos (Pérez 2014, p. 37) racionalizando roles económicos para ambos sexos en la esfera doméstica y extradoméstica y justificando el expolio del medio natural sobre la base de la idea de progreso y de la falacia del crecimiento económico sin fin.

\section{Situando la economía feminista}

En la décadas de los años 60 y 70 del siglo XX, en un contexto de auge de los movimientos sociales, surge la denominada segunda ola del feminismo. A diferencia de la primera ola, que enfatizaba en la superación de obstáculos legales y en la búsqueda de la igualdad a través del sufragio femenino o de los derechos de propiedad, en esta etapa se redefinen conceptos claves para el análisis feminista. Diversas autoras subrayan que son las estructuras económicas y sociales las que subordinan a las mujeres por razón de sexo, de ahí que clamen por una transformación de las relaciones de poder tanto en la esfera pública como en el ámbito privado. Sus aportaciones en el terreno de la sexualidad y de los derechos de reproducción (Cabrera y Escobar 2014, p. 217) fueron fundamentales para su empoderamiento en torno al placer sexual y el control de sus cuerpos.

La Economía feminista surge a la estela de estas reivindicaciones. Un hito de gran importancia, en este sentido, es la Conferencia anual de la American Economic Association en 1992 donde se incorpora por primera vez un panel vinculado directamente con el análisis feminista en la economía (Ferber y Nelson 1993). El impacto de esta nueva corriente se refleja en la creación de la lnternational Association of Feminist Economics (IAFFE) en 1992, y la publicación

4 La división sexual del trabajo es producto de la organización social de la modernidad, donde, a la par que se constituye el contrato social entre los varones sobre las democracias modernas, se crea lo que Carole Pateman (1988) denomina el contrato sexual. Dicho contrato subordina a la mujer a la autoridad del varón y la relega a las tareas reproductivas bajo el modelo de la familia nuclear tradicional y el matrimonio heterosexual.

5 Thomas Malthus, John Stuart Mill y David Ricardo son algunos de estos pensadores que expresan su preocupación por la existencia de límites al crecimiento.

6 "Se utiliza el rótulo de extractivismo en sentido amplio para las actividades que remueven grandes volúmenes de recursos naturales, que no son procesados (o que lo son limitadamente) y pasan a ser exportados" (Eduardo Gudynas 2009, en Acosta 2013, p. 140). 
de la revista Feminist Economics (Benería 1999, p. 60), en la que, por primera vez, se abordan debates en torno a la economía feminista.

La economía feminista es una corriente crítica de pensamiento económico que pretende deconstruir la mirada androcéntrica de la visión dominante e incorporar ciertas dimensiones en el marco de análisis. Si bien, hay que establecer que aún está en proceso de conformar una visión alternativa Conviene distinguir esta escuela de pensamiento de la denominada economía del género. ${ }^{7}$ Esta última no rompe con el sistema capitalista, sino que analiza las desigualdades entre hombres y mujeres dentro del marco del discurso neoclásico. Por tanto lo que pretende es incorporar a las mujeres como objeto de estudio en los marcos teóricos ya establecidos, buscando la igualdad de oportunidades para ambos sexos en la esfera monetizada sin cuestionar el trabajo no remunerado realizado en los hogares.

La "economía feminista", por su parte, tiene como objetivo romper con los paradigmas androcéntricos dominantes, aunque se pueden observar distintas tendencias en función del grado de ruptura planteado (Carrasco 2006, p. 55). En este sentido, se puede hablar de "economía feminista integradora" ${ }^{8}$ de "economía feminista de la ruptura". La primera pretende visibilizar el lado oculto, es decir, el trabajo realizado en los hogares, sacando al ámbito monetizado las esferas invisibles de la economía. Su propuesta se basa en la corresponsabilidad de las tareas realizadas por ambos sexos tanto en la esfera doméstica como en la esfera extradoméstica, para poner fin a la división sexual del trabajo. Sin embargo no cuestiona ni la división públicoprivada de la economía, ni los valores androcéntricos que rigen el sistema.

La "economía feminista de la ruptura" parte de la existencia de un conflicto entre el capital y la vida, es decir, de un conflicto social entre la lógica del beneficio del mercado y la lógica de la sostenibilidad de la vida (Agenjo 2011, p. 85). Esta mirada rompe conceptual, metodológica y políticamente con el corsé del pensamiento dualista y masculinizado del sistema socioeconómico, y establece que el objetivo no puede ser reformar el sistema actual porque está pervertido en múltiples sentidos. Así, las propias estructuras capitalistas delegan el mantenimiento de la vida a las esferas invisibilizadas, privatizadas y feminizadas, en un sistema en el que la vida se encuentra siempre bajo amenaza salvo las pocas dignas de ser rescatadas por el resto de la sociedad (Pérez 2014, p. 53).

Pese a las diferencias entre los distintos enfoques, la "economía feminista" presenta una serie de elementos comunes. Todos ellos parten de la existencia y necesaria transformación de unas relaciones de género que provocan unas relaciones asimétricas de poder en el ámbito social, político y económico. También comparten la idea de que hay que ampliar el concepto de economía para incluir en el análisis los trabajos de cuidados realizados por las mujeres en el ámbito no monetizado de la economía. Por cuidados se entiende el mantenimiento diario de la vida, con sus facetas material y afectiva indisolublemente ligadas e imprescindible para todas las personas (Burns 2007, p. 61).

\section{Situando la economía ecológica}

La relación entre economía y ecología no es nueva. En realidad, la actividad económica no puede existir sin el sustrato biofísico que la sostiene. El interés reciente de la conciencia económica por las cuestiones ecológicas es la consecuencia lógica de la separación del proceso productivo de su base natural a partir de la obra de Ricardo y Marx (Naredo 1987), que ha justificado una organización económica y unas decisiones de política económica que amenazan hoy la misma supervivencia (Martínez Alier y Schlüpmann 1991).

La historia del pensamiento económico pone de manifiesto cómo la ciencia económica se consolidó dejando de lado la realidad física y social en la que transcurre la vida de los hombres.

7 Véanse los trabajos de Pérez (2005) y Picchio (2005) para profundizar en la distinción entre estos dos enfoques.

8 También se denomina economía feminista de la conciliación.

9 Estas ideas se encuentran ampliamente desarrolladas en los trabajos de Carrasco (2001), Pérez (2005, 2014) y Picchio $(1999,2005)$. 
Este alejamiento se refleja en dos aspectos básicos: la limitación del universo del sistema económico a los valores pecuniarios o de cambio y la reducción del concepto de riqueza, objeto de estudio de la economía, a una única categoría de la misma: el capital.

En el interesante libro de Naredo $(1987)^{10}$, La economía en evolución, se expone detalladamente el viraje de la economía hasta constituirse como una ciencia mecanicista, reduccionista y alejada de los valores morales y del contexto físico y natural.

Es cierto que a partir de la década de los sesenta algunos economistas comienzan a ocuparse de modo sistemático de los impactos de las actividades económicas sobre los ecosistemas, y que la "crisis del petróleo" despertó un interés inusitado por el estudio de los aspectos energéticos de los procesos económicos. Sin embargo, inferir de ello que la preocupación económica por los recursos naturales y el medio ambiente debe situarse en fechas tan recientes, muestra no sólo un desconocimiento preocupante (o una visión sesgada) de la historia del pensamiento económico, sino hasta qué punto los economistas se han impregnado de la concepción crematística al uso del proceso económico.

La primera escuela económica que formalizó científicamente la economía fue, a juicio de la mayoría de los autores, la escuela fisiocrática, a mediados del siglo XVIII. La imagen de la economía que surge del enfoque del Tableau économique de Quesnay, es la de una actividad gobernada por leyes naturales. Erigían a la tierra como fuente de riqueza y a la agricultura como único sector productivo. Su "modelo" tiene coherencia hoy día, si se realiza en términos energéticos, algo que no era posible en su tiempo.

Sin embargo, los autores clásicos realizan un desplazamiento de la problemática a la que se enfrentaba la ciencia económica, ahora el centro de interés aparece desvinculado del contexto físico para entrar en la esfera de lo social, al considerar el trabajo como fuente de riqueza. Smith, Ricardo, Malthus discuten sobre la renta de la tierra, las fuentes de riqueza y el valor.

Ya con estos autores se limitó el concepto de riqueza a las cosas útiles que tienen valor de cambio, por lo que se justificó la producción de valores de cambio como único modo de creación de riqueza. Los recursos naturales quedaban fuera del objeto de la economía por varios motivos. Aparentemente no eran un recurso escaso (aunque ya se preocuparon del tema de los rendimientos decrecientes). Además en el caso de su posible agotamiento, existía la fe de su sustitución por otros factores productivos gracias al progreso técnico (autores neoclásicos). Según Naredo, el mito de la máquina creadora de riqueza se extendió hasta considerar a la naturaleza como una máquina ya construida y por tanto gratuita.

Aunque la crítica ecológica de la economía convencional comenzó hace más de cien años y está representada actualmente, entre otros autores, por Georgescu-Roegen ${ }^{11}$, Daly ${ }^{12}$, $\operatorname{set}^{13}, K$ Kapp $^{14}$, etc. (Martínez Alier y Schlüpmann 1991) en realidad la economía ecológica, junto con la economía feminista, está en proceso de conformar una visión alternativa verdaderamente sistemática. En este sentido, la economía ecológica tiene muy poco que ver con la llamada economía ambiental. Esta última intenta valorar los recursos y los efectos ambientales del proceso económico mediante la valoración monetaria de éstos, algo complicado teniendo en cuenta que la metodología económica se redujo, por parte de los neoclásicos, al universo de los objetos apropiables e intercambiables, características que muchos recursos naturales no cumplen. Otras limitaciones que encuentra dicha disciplina para hacer frente a los problemas del medio ambiente es la de valorar las preferencias de las generaciones futuras, así como a la hora de intentar reformar la contabilidad nacional para incluir el capital natural dentro del capital o riqueza na-

\footnotetext{
${ }^{10}$ Véase Naredo, J. M. 1996 [1987]. La economía en evolución. Madrid, Siglo XXI de España.

${ }^{11}$ Véase Georgescu-Roegen, N. 1996 [1971]. La Ley de la Entropía y el Proceso Económico. Madrid, Fundación Argentaria-Visor distribuciones..

${ }^{12}$ Véase Daly, Herman E. 1989 [1980]: Economía, Ecología, Ética: ensayos hacia una economía en estado estacionario. México D.F., Fondo de Cultura Económica.

${ }^{13}$ Véase Passet, R. 1996 [1979]: Principios de Bioeconomía. Madrid, Fundación Argentaria-Visor distribuciones.

${ }^{14}$ Véase Kapp, K.W. 1978. El carácter de sistema abierto de la economía y sus implicaciones, La economía del futuro, Doepfer, K. (ed.), México, FCE, pp. 126-146.
} 
cional. En definitiva, la imposibilidad de valoración monetaria y de apropiación, hacen difícil incluir a la naturaleza dentro de estas metodologías.

Mientras que la economía ambiental constituye más bien una especialización de la economía neoclásica, la economía ecológica se reclama el objetivo de un enfoque "ecointegrador" cuyos fundamentos "afectarían al método, al instrumental e incluso al propio estatuto de la economía, al sacarla del universo aislado de los valores de cambio en el que hoy se desenvuelve para hacer de ella una disciplina obligadamente transdisciplinar" (Naredo 2006).

Desde el punto de vista de la economía ecológica, tratar de extender el ámbito de lo económico al campo de los recursos naturales, exige abandonar los principios, clasificaciones y conceptos sobre los que se levanta la abstracción de sistema económico en la actualidad. Si se desea adaptar la gestión de los recursos a las características del entorno, con vistas a evitar su degradación, no cabe partir de una valoración puntual e incompleta de algunos de sus componentes, atendiendo a criterios de la subjetividad humana, sino preocuparse de analizar las características intrínsecas de ese entorno y enjuiciar el papel que desempeña cada una de sus partes en el mantenimiento de la biosfera y de la vida humana.

Sería injusto atribuir única y exclusivamente a la línea de pensamiento que surge en el campo de la economía ecológica el mérito de reclamar un enfoque sistémico para la economía. El enfoque sistémico en economía hunde sus raíces en aquella visión reproductiva de los procesos económicos de la que surge, precisamente, tanto la necesidad de considerar el sustrato bio-físico de la actividad económica, como la de verificar la consistencia de los resultados obtenidos con las ciencias adyacentes, la ecología incluida (Barceló y Ovejero 1985). Como ha señalado el mismo Barceló (1992, p. 83): "En última instancia, el mérito del enfoque reproductivo radica, a nuestro entender, no en consideraciones apriorísticas, sino en el hecho físico e histórico de que en el planeta Tierra la vida material de la especie humana se articula mediante procesos de producción y reproducción de bienes y de relaciones de todo tipo, con un marco natural sometido a ciclos periódicos. La estrecha compatibilidad del enfoque reproductivo en economía y del enfoque ecosistémico en ecología son, en este sentido, indicios estimables de corrección científica".

Los pioneros en la economía ecológica son autores dentro del campo de las ciencias sociales y naturales del siglo XIX y principios del siglo XX que se plantearon ciertos aspectos de la relación entre sistema económico y flujos de energía en la sociedad humana, una vez formuladas las leyes de la termodinámica. Algunos de los más destacados son Sergei Podolinsky (1850$1891)^{15}$; Patrick Geddes (1854-1932) ${ }^{16}$; Frederick Soddy $(1877-1956)^{17}$; Nicholas GeorgescuRoegen (1906-1994) ${ }^{18}$ y su libro clave: La Ley de la entropía y el proceso económico (1971);

\footnotetext{
${ }^{15}$ Sergei Podolinsky (1850-1891), un médico socialista ucraniano que se encargó de estudiar la actividad humana como un sistema de conversión energética, estudiando los ratios input-output de la agricultura en términos energéticos, en la década de los 80 del siglo XIX.

${ }^{16}$ Patrick Geddes (1854-1932), urbanista y planificador regional, desarrolló los principios básicos de una especie de tableau économique en términos físicos y criticó la contabilidad económica porque no seguía el rastro de las pérdidas energéticas y materiales en el proceso económico ni valoraba la contaminación. También fue uno los primeros autores que trató de interpretar la historia humana en términos de cambios en el uso de la energía.

${ }^{17}$ Frederick Soddy (1877-1956), químico muy conocido. Su idea principal era que los economistas estaban confundiendo el capital real con el capital financiero y criticó las teorías imperantes sobre el crecimiento económico.

${ }^{1818}$ Nicholas Georgescu-Roegen (1906-1994), profesor americano de origen rumano, matemático y economista, fue uno de los primeros autores que propuso, desde dentro de la profesión, trascender el universo del valor en el que la economía se ha desenvuelto desde Adam Smith, para ampliar su objeto de estudio, abriéndolo hacia otros campos de conocimiento y muy particularmente hacia esa economía física que es la termodinámica. Reformuló el núcleo duro de la economía, matemático pretendidamente cuantitativo, proponiendo un auténtico cambio de paradigma. No sólo arremetió contra la función de utilidad sino también contra la función de producción. El objeto de su libro más conocido es precisamente fundamentar la idea de que el estudio del proceso económico debe estar ligado a la Ley de la entropía, lo que le conduce a tratar muchos aspectos fuera del campo de la economía convencional.
} 
René Passet ${ }^{19}$ y el enfoque de este autor patente en su libro Principios de Bioeconomía (1979); Herman E. Daly ${ }^{20}$, discípulo de Roegen que ha desarrollado un concepto sobre la economía en estado estacionario. Actualmente existen muchos seguidores y pensadores de economía ecológica. En España, los más destacados son José Manuel Naredo, Joan Martínez Alier, Federico Aguilera Klink y Vicent Alcántara.

Las nociones biofísicas fundamentales sobre las que se articula la Economía ecológica son tres:

a) La primera consiste en el reconocimiento de la verdad elemental que expresa la Primera Ley de la Termodinámica, según la cual la materia y la energía no se crean ni se destruyen, sino que sólo se transforman. A pesar de que esta Ley se usa para justificar una visión mecánica e irreal de una economía que no agota recursos, la realidad es que permite echar por tierra la noción de externalidades ambientales —entendidas como algo ocasional— puesto que es evidente, de acuerdo con la citada Ley, que la generación de residuos es algo inherente a los procesos de producción y consumo.

b) La segunda es la Ley de la Entropía o Segunda Ley de la Termodinámica, que apunta que la materia y la energía se degradan continua e irrevocablemente desde una forma disponible a una forma no disponible, o de una forma ordenada a una forma desordenada, independientemente de que las usemos o no. Así pues y desde el punto de vista de la termodinámica, lo que confiere valor económico a la materia y energía es su disponibilidad para ser utilizada, por contraste con la energía y materia no disponible o ya utilizada, a la que debemos considerar como residuo en un sentido termodinámico (Georgescu-Roegen 1996).

c) La tercera noción presenta una doble vertiente. La primera de ellas se refiere a la imposibilidad de generar más residuos de los que puede tolerar la capacidad de asimilación de los ecosistemas, so pena de destrucción de los mismos y de la vida humana. La segunda advierte de la imposibilidad de extraer de los sistemas biológicos más de lo que se puede considerar como su rendimiento sostenible o renovable (Daly 1991) pues de lo contrario acabaríamos con ellos e, indirectamente, con nosotros mismos. Todo esto exige un conocimiento profundo de la estructura y funcionamiento de los ecosistemas naturales, que son la base de la vida humana y de las sociedades, conocimiento que marca los límites, tanto físicos como conceptuales, a los que debe ajustarse la actividad humana y por lo tanto la economía (Farras 1980) .

En definitiva, los fundamentos biofísicos y la propia ecología nos enseñan que el hombre no utiliza recursos naturales de manera aislada (Toledo 1985), sino que utiliza ecosistemas, proceso de apropiación que ha sido correctamente interpretado por Norgaard (1984) como un proceso coevolucionario. Esto significa que en la medida en la que el sistema socioeconómico modifica los sistemas biológicos, se ve obligado a su vez a adaptar el primero a los cambios introducidos en el segundo, de manera que sea capaz de comprender los efectos de las modificaciones sobre los ecosistemas que le permita usar adecuadamente los mismos, para lo cual necesita crear nuevas instituciones, en el sentido de nuevas leyes, reglas o normas sociales de comportamiento. De ahí la necesidad imperiosa de contar con una "ecología social" (Bookchin 1978) que investigue los factores económicos e institucionales involucrados en la crisis ambiental actual y en sus posibles soluciones.

\footnotetext{
${ }^{19}$ René Passet. El enfoque de este autor patente en su libro Principios de Bioeconomía (primera edición en francés de 1979), le lleva a tratar el tema de la energía junto con el de la información. Apreciando que la vida deriva parte de la energía disipada hacia la construcción y el mantenimiento de estructuras complejas, Passet considera por analogía el proceso económico como proceso de "distribución creadora". Subraya el creciente peso de los aspectos inmateriales en la producción de valor, ejemplificado por la expansión del mundo financiero a tasas muy superiores a las de la llamada "economía real".

${ }^{20}$ Herman E. Daly ha desarrollado un concepto sobre la economía en estado estacionario, definido por la combinación del equilibrio biofísico y el crecimiento moral, cuya consecución sería a través de mantener un acervo constante de riqueza física y un acervo constante de personas, con una baja tasa de sustitución de estos elementos (tanto en términos de materia como de energía).
} 
Por otro lado, reconocer que la unidad de gestión apropiada es el ecosistema y no el recurso, cuestiona la noción de propiedad privada, en su acepción ideológica más extendida y estricta, así como la solución de recurrir a la privatización del medio ambiente puesto que "el ejercicio total de la propiedad privada es virtualmente imposible en un contexto de ecosistemas" (Regier et al. 1989, p. 114). Dentro del marco institucional o legal cuyo objetivo sea la gestión del Planeta y el acceso equitativo a la renta y la riqueza, es necesario dejar de contemplar los derechos privados como derechos absolutos e independientes de un fin.

Dicho de otra manera, hay que reconocer que "todos los derechos (de propiedad) son condicionales y derivativos; derivan del fin o del objetivo de la sociedad en que se dan; están condicionados a que se los use para contribuir al logro de ese fin, no para obstaculizarlo" (Tawney 1972, p. 51). Está claro que el fin, en este caso, es el mantenimiento de la vida en un contexto de libertad; lo contrario sería apoyar el ecofascismo. Por eso es realmente significativo que la CEE en su Quinto Programa Ambiental manifestara por primera vez la necesidad de que los ciudadanos europeos cambien sus pautas de comportamiento y de consumo, es decir, su estilo de vida. Cambio cuya iniciativa no la toma el mercado, sino que va a estar orientado mediante decisiones políticas, algunas de las cuales se van a aplicar poniendo el mercado al servicio de la sociedad y no la sociedad al servicio del mercado.

La mayoría de las ideas apuntadas no son en absoluto nuevas. Algunas se remontan a Aristóteles, otras son más recientes y pertenecen al pensamiento de las ciencias sociales y naturales del siglo XIX y principios del XX (Martínez Alier y Schlüpmann 1991). Dos de los principales problemas para su profundización y difusión, dejando aparte los importantes intereses económicos que hay en juego, son la resistencia (Georgescu-Roegen 1971) a considerar la economía como algo diferente de la mecánica, y la resistencia al trabajo transdisciplinar.

\section{Encuentros entre economía feminista y economía ecológica}

Como ya hemos mencionado anteriormente, el proceso de acumulación capitalista se apoya en la explotación de los recursos naturales y en el trabajo gratuito realizado por las mujeres en los hogares. Sin embargo, el discurso económico dominante ignora, de forma sistemática, estas dos dimensiones fundamentales del proceso de acumulación. También se han expuesto las líneas maestras de dos discursos críticos y alternativos, que surgen de la preocupación por incluir en el análisis estas dos dimensiones: la "economía feminista" y la "economía ecológica".

En este epígrafe se van a buscar los puntos de encuentro entre estas dos corrientes. El primero a destacar es que ambas corrientes sitúan la sostenibilidad de la vida, en su dimensión humana, social y ecológica, en el centro del análisis (Pérez 2005, p. 159). Dicha sostenibilidad se entiende como relación armónica de los seres humanos y la naturaleza, y como relaciones de equidad entre las propias personas. Se trata de un proceso que no sólo busca la posibilidad de que la vida continúe, sino que, además, pretende desarrollar niveles de vida aceptables para toda la población (Bosch et al. 2005, p. 322).

El segundo aspecto en común se refiere a que tanto la economía feminista como la ecológica comparten la idea de que los servicios de la naturaleza y los trabajos invisibles son esenciales para la satisfacción de las necesidades. Por necesidades humanas se entiende todas aquellas necesidades básicas, fisiológicas y afectivas, que deben de ser satisfechas para preservar la propia integridad de la persona. Ello requiere la existencia de unas condiciones ambientales favorables para que exista la vida humana en el planeta. Ambos enfoques críticos comparten la teoría de las necesidades humanas propuesta por el economista Max-Neef, que desagrega las necesidades en categorías existenciales y axiológicas. Esta combinación permite operar con una clasificación que incluye, por una parte, las necesidades de Ser, Tener, Hacer y Estar; y, por la otra, las necesidades de Subsistencia, Protección, Afecto, Entendimiento, Participación, Ocio, Creación, Identidad y Libertad (Max-Neef 1998, p. 41). Por tanto, se entiende que los trabajos de cuidados realizados por las mujeres en el hogar están relacionados no sólo con alimento y vestido, sino con otros aspectos emocionales y afectivos (Bosch et al. 2005, p. 324).

El tercer punto de encuentro entre ambas corrientes críticas es el relativo a su confrontación con la estructura de pensamiento occidental sustentada en la supremacía del hombre y la subordinación del género femenino y del medio natural. Esta estructura de pensamiento, denominada 
eurocentrismo, se basa en una serie de dicotomías universalizables, tales como cultura/naturaleza, masculino/femenino, moderno/tradicional, público/privado, razón/emoción, europeo/no europeo, pretende convertirse en perspectiva globalmente hegemónica y desplazar los "modos de conocer" de otras culturas. En esta serie sin fin de dualismos enfrentados, una parte encarna la normalidad frente a su opuesto que debe ser controlado. Mujer y naturaleza se encuentran en la parte invisible, anormal y desviada en dichas dicotomías, lo que justifica ideológicamente su control y explotación.

La epistemología hegemónica y patriarcal reacciona mediante la violencia simbólica naturalizada contra todo lo que se encuentre al margen del terrero de la producción y del sujeto masculino europeo.

Ambas posiciones críticas también proponen ampliar la esfera de lo económico más allá del ámbito de lo monetario, y concebir a la naturaleza y a los trabajos no remunerados no sólo como parte de la economía sino como aspectos clave del proceso económico (Pérez 2014, p. 24). Como se ha comentado, el modelo económico actual deja fuera del mundo económico los trabajos de cuidados realizados por las mujeres y la explotación de los recursos naturales, porque los concibe como actividades no remuneradas a precios de mercado (Bosch et al. 2005, p. 333). La economía ecológica y la economía feminista conciben a la economía como un subsistema abierto y a las familias como reproductoras de la fuerza de trabajo necesaria para la continuidad del propio sistema más que como simples unidades de consumo.

El cuarto punto de encuentro se relaciona con la existencia de una voraz crisis ecológica y de cuidados en base a una falsa idea de autonomía del sistema económico (Bosch et al. 2005, p. 336). Para ambas corrientes, los seres humanos somos doblemente dependientes: ecodependientes de un planeta con unos recursos naturales finitos, e interdependientes de los cuidados y los afectos de otras personas. Para la economía feminista y la ecológica existen una deuda ecológica y una deuda de cuidados. La primera es la que los países ricos tienen con los países empobrecidos debido al desigual uso de los recursos y bienes naturales, así como la desigual responsabilidad en el deterioro y destrucción del medio físico (Herrero 2008, p. 15). La segunda es la contraída por los hombres con las mujeres de todo el mundo por el trabajo que realizan y han realizado gratuitamente para la reproducción de la vida. La entrada masiva de las mujeres en el mundo laboral produjo una transformación de las estructuras tradicionales que sustentaban los cuidados realizados el hogar, provocando la duplicación de la jornada laboral femenina en la esfera doméstica y en la extradoméstica.

Por último, merece destacarse que la economía feminista y la economía ecológica cuestionan la concepción del tiempo del pensamiento económico dominante. Éste tiene una visión de la distribución del tiempo muy limitada, dado que lo divide en tiempo dedicado a la esfera pública o tiempo industrial y tiempo de ocio. El primero es un tiempo-reloj, una construcción cultural en consonancia con la manera de entender el sistema socioeconómico ${ }^{21}$. El tiempo se mercantiliza, se transforma en un tiempo cronométrico descontextualizado en el que todas las horas son secuenciales y lineales. Todo esto está íntimamente ligado al concepto de trabajo de la economía capitalista, reducido a la idea de empleo, de forma que toda actividad que no tenga fijado un valor monetario queda fuera del ámbito de la producción. Según Legarreta (2013), la secuencia es la siguiente: el trabajo se reduce a actividad, la actividad a tiempo y el tiempo a dinero. Y como el dinero es un medio cuantitativo, el tiempo que se intercambia por dinero ha de ser, inexorablemente, de tipo cuantitativo.

Sin embargo, existe otra idea de tiempo relacionado con la lógica de la reciprocidad, un tiempo multidimensional que tiene en cuenta el ritmo ecológico de la naturaleza y el período biológico del cuerpo. Por una parte, los ecosistemas y los diversos seres vivos que habitan en ellos tienen unos procesos evolutivos y unos ciclos vitales, sin embargo la voracidad por acumular el

\footnotetext{
21 "La concepción del tiempo no es a-histórica ni universal, sino que revela un desarrollo histórico concreto y contextualizado: se generaliza a lo largo del siglo XIX junto con los procesos de industrialización y urbanización, y responde a una concepción mercantilista del trabajo y de la economía” (Legarreta 2013)
} 
capital no respeta los ritmos naturales de estos espacios superando los límites de sustentabilidad del planeta. Por otra, hay una dimensión del tiempo, ignorada por los ritmos que marca el modelo económico, unido a la corporalidad. Así, no sólo hacemos referencia al tiempo biológico de las necesidades humanas, sino también a la dimensión más subjetiva que incluye los cuidados, los afectos, en definitiva, las necesidades emocionales. De esta manera la producción capitalista está continuamente violentando todo lo que signifique tiempos de vida, y de esa manera atenta contra la naturaleza como sistema ecológico y contra las mujeres como sostenedoras de vida (Bosch et al. 2005, p. 343).

\section{Una mirada conjunta hacia el paradigma de la sostenibilidad de la vida: ecofeminismo}

Como venimos comentando en este artículo, existe una irreconciliable contradicción entre el proceso de reproducción natural y social y el proceso de acumulación de capital. El deterioro social y ambiental es consecuencia de un modelo de desarrollo basado en el crecimiento ilimitado y, por otro lado, la consideración de los mercados como epicentro de la sociedad impide el mantenimiento de la vida humana en condiciones dignas. La perspectiva ecológica demuestra la inviabilidad física de la sociedad del crecimiento y el enfoque feminista denuncia la lógica de la acumulación y el crecimiento como una lógica patriarcal y androcéntrica, de forma que la tensión entre el capitalismo y la sostenibilidad humana y ecológica muestra la oposición esencial entre el capital y la vida.

Resulta evidente la necesidad de un cambio de paradigma, demandado por muchos movimientos sociales alternativos, que reclaman un cambio radical del sistema civilizatorio actual, para asegurar el bienestar social de las poblaciones actuales y de las generaciones futuras. Para ello es preciso realizar una revisión profunda que permita indagar por dónde deben caminar los procesos económicos y sociales para ser compatibles con los ciclos naturales. Se debe mostrar que la concepción de progreso adoptada por la humanidad está muy ligada al deterioro ecológico; que el individualismo y la propiedad privada no son "naturales" y que, a lo largo de la historia la naturaleza y los seres humanos, especialmente las mujeres, han adoptado estrategias colectivas de cooperación (Naredo 2006).

En este trabajo, se propone colocar la satisfacción de las necesidades y el bienestar de las personas en condiciones de equidad como objetivo del progreso social y económico, como eje vertebrador de la sociedad, y por tanto, de los análisis. Para orientar este cambio de paradigma y colocar la supervivencia individual y colectiva en el centro de nuestras miradas y de la política, es imprescindible valorar los trabajos que el mercado ignora (Herrero 2006).

Alcanzar la sostenibilidad obliga a que las sociedades se hagan responsables de la vida. Supone reducir las extracciones de materiales finitos, disminuir la generación de residuos y conservar los equilibrios de los ecosistemas. Se precisa analizar qué trabajos son social y ambientalmente necesarios y cuáles sería deseable descartar, de forma que se valore en qué medida facilitan el mantenimiento de la vida en términos de equidad. Por tanto, el enfoque de la sostenibilidad está ligado a otra forma de orientar el mundo del trabajo y diferenciar entre aquellos ligados a la producción o a la destrucción de la vida. Además es preciso modificar el modelo de división sexual del trabajo y realizar cambios drásticos en los espacios domésticos de forma que se corresponsabilice tanto a hombres como mujeres en las tareas de mantenimiento de la vida (Herrero 2006).

Desde esta perspectiva se sostiene que el feminismo y el ecologismo están llamados a enriquecerse mutuamente, y tendrán un papel fundamental en la construcción de nuevas formas de entender el mundo y hacer frente a los retos que se plantean en nuestro siglo. El ecofeminismo surge como una filosofía y práctica feminista y plantea alternativas a la crisis de valores de la sociedad individualista y consumista actual. Las aportaciones de ambas perspectivas críticas feminista y ecologista- ofrecen la oportunidad de enfrentar el sexismo de la sociedad patriarcal al tiempo que denuncia el sesgo androcéntrico de la cultura occidental dominante, basada en la dominación de la naturaleza (Puleo 2011).

Los orígenes teóricos se sitúan en la década de los setenta, con la publicación de "Feminismo o la muerte" de Françoise D’Eaubourne, donde aparece por primera vez el término. Existen numerosas propuestas ecofeministas, pero todas comparten la visión de que la subordinación de 
las mujeres a los hombres y la explotación de la naturaleza son dos fenómenos que responden a la lógica de la dominación y el desprecio a la vida. Los planteamientos ecofeministas someten a revisión conceptos claves de nuestra cultura dominante como la modernidad, y la forma de entender el progreso, puesto que han mostrado su incapacidad para lograr el bienestar humano y proporcionar a las personas una vida digna (Puleo 2011).

Dada la novedad de sus planteamientos y por ser una de las formas más recientes del feminismo, el enfoque ecofeminista suele ser poco y mal conocido y rechazado bajo el calificativo de "esencialista". Sin embargo, cabe resaltar que esta perspectiva plantea que es la división sexual del trabajo y la distribución del poder y la propiedad, la que ha sometido a las mujeres y al medio natural del que todos los seres humanos formamos parte. Trata de hacer visible el sometimiento, y señalar las responsabilidades en nombre de la justicia social y ambiental, y corresponsabilizar a hombres y mujeres en el trabajo de la supervivencia. Plantea que la alternativa pasa por reconstruir una nueva cultura que convierte en visible la ecodependencia para todos los seres humanos, señalando que no es posible la sostenibilidad si no se asume la equidad de género (Puleo 2011).

En definitiva, nuestra conciencia como especie humana ha de avanzar hacia la igualdad de hombres y mujeres, en tanto partícipes no sólo de la cultura, sino también de la naturaleza. La cultura del cuidado tendrá que ser rescatada si queremos llegar a formas de organización social y ecológicamente sostenibles, que permitan alcanzar la igualdad y la ecojusticia, o simplemente para sobrevivir como especie (Shiva 2006).

\section{Reflexiones}

Como venimos advirtiendo a lo largo del artículo, la teoría económica dominante se articula en torno a un modelo con un patrón de acumulación que sitúa a los mercados en el centro de toda la estructura socioeconómica, e ignora las dimensiones ecológicas y de género. En este trabajo se parte de la necesidad de deconstruir dicho modelo e incorporar nuevas dimensiones de análisis y marcos teóricos que tiendan hacia un paradigma alternativo. Para transitar hacia un nuevo modelo económico que garantice la producción y la reproducción de la vida humana y no humana, se asumen el enfoque de la economía feminista y de la economía ecológica que contemplan, a nuestro entender, dimensiones claves para garantizar una vida en armonía con los ecosistemas.

En primer lugar se reivindica desde ambas posiciones críticas la necesidad de reconocer la existencia del conflicto que enfrenta el capital con la vida bajo un ideal antropocéntrico de autosuficiencia que niega la interdependencia, vulnerabilidad y ecodependencia del ser humano.

En segundo lugar se apuesta por un proyecto que coloque la vida en el centro del sistema socioeconómico, que acepte los límites del planeta y que niegue el ideal de autosuficiencia de los sujetos. Esto implica tener en cuenta dos cuestiones principales: por un lado, mantener un principio de prudencia en cuanto a la utilización de los recursos materiales y energéticos, que respete la justicia ambiental. Por otro, la necesidad de visibilizar la lógica del cuidado y respetar la singularidad de los cuerpos dinamitando los roles económicos asociados a la masculinidad y feminidad.

En último lugar, se propone el ecofeminismo como uno de los posibles caminos para construir otro paradigma que sitúe la sostenibilidad de la vida en el centro. En este enfoque se encuentran las miradas ecologista y feminista al tener en cuenta las dos dimensiones claves a incorporar en el marco analítico, el trabajo realizado por las mujeres en el ámbito doméstico y los servicios prestados por la naturaleza.

\section{Bibliografía}

Acosta, Alberto. 2010. El Buen Vivir en el camino del post-desarrollo. Una lectura desde la Constitución de Monstecristi. Octubre. http://www.rebelion.org/docs/118561.pdf

- 2013. El Buen Vivir: Sumak Kawsay, una oportunidad para imaginar otrosmundos. Barcelona, Icaria Editorial. 
Agenjo, Astrid. 2011. Lecturas de la crisis en clave feminista: una comparación de la literatura en torno a los efectos específicos sobre las mujeres, Papeles de Europa, 23, 70-100.

Amoroso, María Inés; Bosch, Ana y Fernández, Hortensia. 2003. Malabaristas de la vida. Barcelona, Icaria Editorial.

Barceló, Alfons.1992. Filosofía de la economía. Leyes, teorías y modelos. Barcelona, Icaria/Fuhem.

Barceló, Alfons y Ovejero, Félix. 1985. Cuatro temas de metodología económica, Cuadernos de Economía, 13 (37), 215-248.

Benería, Lourdes. 1999. El debate inconcluso sobre el trabajo no remunerado, Revista Internacional del Trabajo, 3,321-346.

Bookchin, Murray. 1993. Por una sociedad ecológica. Barcelona, Gili.

Bosch, Anna; Carrasco, Cristina y Grau, Elena. 2005. Verde que te quiero violeta. Encuentros y desencuentros entre feminismo y ecologismo. En Enric Tello, La historia cuenta. Del crecimiento económico al desarrollo humano sostenible. España, Ediciones de Intervención Cultural/El Viejo Topo.

Burns, Alison Teresa. 2007. Politizando la pobreza: hacia una economía solidaria del progreso. El Salvador, Instituto de Investigación Capacitación y Desarrollo de la Mujer IMU.

Cabrera, Mary y Escobar, Lorena. 2010. Equidad de género y economía social y solidaria: Aportes de la economía feminista. En Yolanda Jubeto, Luis Guridi y Maite Fernández (eds.) Diálogos sobre Economía Social y Solidaria en Ecuador. Encuentros y Desencuentros con las propuestas para otra economía. Bilbao, Universidad del País Vasco e Instituto de Estudios sobre Desarrollo y Cooperación Internacional.

Carrasco, Cristina. 2001. La sostenibilidad de la vida humana: ¿un asunto de mujeres?, Revista Mientras Tanto, 82, Barcelona, Icaria.

- 2006. La economía feminista: Una apuesta por otra economía. En María Jesús Vara (coord.) Estudios sobre Género y Economía. Madrid, Akal, pp. 29-62.

- 2012. Economía, trabajos y sostenibilidad de la vida. En Red de Economía Solidaria y Alternativa Euskadi, Sostenibilidad de la vida. Aportaciones desde la Economía Solidaria, Feminista y Ecológica. Bilbao, Universidad del País Vasco.

- 2014. Con voz propia: La economía feminista como apuesta teórica y política. Cristina Carrasco(ed.). Madrid, La Oveja Roja.

Daly, Herman E. 1990. Toward some operational principles of sustainable development, Ecological Economics, 2 (1), 1-6.

Farras, A. 1980. Bases ecológicas para una aproximación a la problemática del medio Ambiente, Mientras Tanto, 5, 58-70.

Ferber, Marianne y Nelson Julie. 1993. Introduction: the social construction of economics and the social construction of gender. En Marianne Ferber y Julie Nelson (eds.) Economic Man: Feminist Theory and Economics. Chicago, University of Chicago Press.

Gallego, Elena. 2000. Un lugar para Jane Marcet y Harriet Martineau dentro de la escuela clásica, ICE Revista Historia y Pensamiento Económico, 789, 101-104.

- 2010. La incorporación de las mujeres a la economía política en los albores del Siglo XIX. Revista ICE Historia y Pensamiento Económico, 852, 13-25.

- 2011. El compromiso social en los Principios de Economía Política y en la Sujeción de las mujeres de Jonh Stuart Mill (con las contribuciones de Harriet Taylor Mill),Revista de la Asociación Española de Historia Económica, 19, 59-84.

Georgescu-Roegen, Nicholas. [1975] 1996. La Ley de la Entropía y el proceso económico. Madrid, Fundación Argentaria.

Herrero, Yayo. 2006. Ecofeminismo: una propuesta de transformación para un mundo que agoniza, Cuadernos Mujer y Cooperativismo, 8, 74-80 PUCMTA.

- 2008. Tejer la vida en verde y violeta. Vínculos entre ecologismo y feminismo,Cuadernos de Ecologistas en Acción, 13, 2-23.

- 2012. Propuestas ecofeministas para un sistema cargado de deudas, Revista de Economía Crítica, 13 (1), 30-54. 
Legarreta, Iza. 2013. El tiempo como herramienta para la economía feminista. Una propuesta a partir del estudio del trabajo doméstico y los cuidados. Sevilla, IV Jornadas de Economía Feminista, Universidad Pablo de Olavide. Octubre.

Martínez Alier, Joan y Schlüpmann, Klaus (1991), La ecología y la economía. México, FCE.

Mayordomo, Maribel. 2000. Precursores: el trabajo de las mujeres y la Economía Política. Albacete, VII Jornadas de Economía Crítica, Universidad de Castilla la Mancha. Febrero.

Naredo, José Manuel. 1987. La economía en evolución. Madrid, Siglo XXI.

- 2006. Raíces económicas del deterioro ecológico y social. Más allá de los dogmas. Madrid, Siglo XXI.

Neef-Max, Manfred. 1998 [1993]. Desarrollo a Escala Humana. Barcelona, Icaria.

Norgaard, Richard. 1984. Coevolutionary Development Potential, Land Economics, 60(2), 160173.

Passet, René. 1979. Principios de Bioeconomía. Madrid, Fundación Argentaria-Visor distribuciones.

Pateman, Carole. 1995 [1988]. El contrato sexual. Barcelona, Anthropos

Perdices, Luis y Gallego, Elena. 2007. Mujeres Economistas: Las aportaciones de las mujeres a la ciencia económica y su divulgación durante los siglos XIX y XX. Madrid, Ecobook.

Pérez, Amaia. 2005. Perspectivas feministas en torno a la economía: El caso de los cuidados. Tesis doctoral. España, Universidad Complutense de Madrid.

- 2014. La subversión feminista de la economía. Aportes para un debate sobre el conflicto capital-vida. Madrid, Traficantes de Sueños.

Pérez, Laura y Domínguez-Serrano Mónica. 2014. Una reformulación feminista del Decrecimiento y el BuenVivir. Contribuciones para la sostenibilidad de la vida humana y no humana. Valladolid, XIV Jornadas de Economía Crítica, Universidad de Valladolid. Septiembre.

Picchio, Antonella. 1999. Visibilidad analítica y política del trabajo de reproducción social. En Cristina Carrasco (coord.): Mujeres y Economía, Barcelona, Icaria.

- 2005. La economía política y la investigación sobre las condiciones de vida. En Cairós y Mayordomo (comps.), Por una economía sobre la vida, Barcelona, Icaria.

Puleo, Alicia. 2011. Ecofeminismo para otro mundo posible. Madrid, Cátedra.

Regier H. et al.1989. Reforming the Use of Natural Resources. En Common Property Resources. Ecology and Community-Based Sustainable Development, Berkes, F. (Ed.), London, Belhaven Press.

Riechmann, Jorge; Carpintero, Óscar y Matarán Alberto (coords.). 2014. Los inciertos pasos desde aquí hasta allá. Alternativas Socioecológicas y transiciones poscapitalistas. Granada, Periferias, Universidad de Granada.

Shiva, Vandana. 2006. Manifiesto para una Democracia de la Tierra. Justicia, sostenibilidad y paz. Barcelona, Paidós.

Tawney, Richard Henry. 1972. La sociedad adquisitiva. Madrid, Alianza.

Tello, Enric. 2005. La historia cuenta. Del crecimiento económico al desarrollo humano sostenible. España, Ediciones de Intervención Cultural/El Viejo Topo.

Toledo, Victor. 1985. Ecología y Autosuficiencia Alimentaria. México, Siglo XXI. 